\title{
Concepciones alternativas sobre Astronomía de profesorado español en formación
}

\section{Spanish pre-service teachers' alternative conceptions about Astronomy}

\author{
María-Mercedes Varela-Losada ${ }^{1}$ • Uxío Pérez-Rodríguez ${ }^{1}$ • \\ María Álvarez-Lires ${ }^{1}$. Azucena Arias-Correa ${ }^{1}$
}

\begin{abstract}
Resumen: El proceso de enseñanza y aprendizaje de la Astronomía parece entrañar importantes dificultades, particularmente propiciando la generación de concepciones alternativas, como han mostrado numerosas investigaciones procedentes de diferentes contextos. El objetivo de este estudio es indagar sobre concepciones alternativas de profesorado en formación relacionadas con aspectos astronómicos, investigando si en el marco español aparecen los mismos esquemas alternativos que en otros contextos. Los resultados ponen en evidencia que el alumnado mencionado posee un gran número de concepciones alternativas sobre astronomía básica y que estas son similares a las encontradas en otros países.
\end{abstract}

Palabras-clave: Astronomía. Concepciones alternativas. Formación del profesorado. Enseñanza y aprendizaje.

\begin{abstract}
The process of teaching and learning astronomy is often fraught with difficulties. The most frequently reported is the ability to generate alternative conceptions, as shown by numerous investigations in different contexts. The objective of this study is to approach the most common alternative conceptions of pre-service teachers, and also to check whether the Spanish alternative conceptions are the same as in other contexts. The results show that these students also held a number of alternative conceptions about basic astronomy and that these are similar to those found in other countries.
\end{abstract}

Keywords: Astronomy. Alternative conceptions. Teacher training. Teaching and learning process.

\footnotetext{
${ }^{1}$ Departamento de Didácticas Especiales, Facultad de Ciencias de la Educación y del Deporte, Universidad de Vigo (UVI), Campus A. Xunqueira, s/n, 36005, Pontevedra, España. E-mail: <mercedesvarela@uvigo.es>
} 


\section{Introducción}

Numerosos estudios (SCHOON, 1995; TRUMPER, 2001; ZEILIK; SCHAU; MATTERN, 1998) señalan que la Astronomía es una disciplina difícil de enseñar y de aprender, y que ello, en buena parte, es debido a que presenta varias características que propician la generación y persistencia de concepciones alternativas por parte del alumnado. La mayor parte de la literatura sobre esta problemática procede de países anglosajones, pero también es un tema de interés actual en el ámbito iberoamericano, como muestran los trabajos de Bretones y Compiani (2011), Iachel, Langhi y Scalvi (2008), Langhi (2011), Langhi y Nardi (2007, 2010) en Brasil, o Gangui, Iglesias y Quinteros (2010) y Kriner (2004) en Argentina. En el contexto español se han realizado diversos estudios sobre esta temática (CAMINO, 1995; GARCÍA; MONDELO; MARTÍNEZ, 1996; NAVARRETE, 1998; VEGA, 2001), la mayoría de los cuales están enfocados hacia concepciones acerca de fenómenos astronómicos muy concretos en alumnado no universitario (MANUEL, 1995; MARTÍNEZ; MARTÍNEZ, 2005; NAVARRETE, 2004; VEGA, 2001, 2002). Por ello, se consideró necesario indagar sobre ideas alternativas de profesorado en formación, relacionadas con aspectos astronómicos diversos, investigando si en el marco español aparecen los mismos esquemas alternativos que en otros contextos.

En la comprensión de los temas astronómicos, una particularidad decisiva es la dificultad que conlleva luchar contra las propias percepciones sensoriales, cuyas interpretaciones inmediatas muchas veces no están en consonancia con las explicaciones propias de la ciencia de las comunidades científicas. Es difícil interiorizar que la Tierra no es el centro del Universo, ya que ello implica lidiar con la visión del espacio desde el propio punto de referencia, o entender que no es correcto pensar que el verano ocurre porque nuestro planeta está más cerca del sol, porque ello contradice las observaciones cotidianas según las cuales cuanto más cerca se halla un foco de calor mayor es la temperatura (SANMARTí, 2003). Tal y como defiende Kriner (2004) la dificultad en la enseñanza de la Astronomía radica en el alto grado de abstracción de los conceptos y del conocimiento espacial que requiere. Así, para comprender las variaciones en la apariencia de la Luna son necesarios ciertos conocimientos sobre el espacio y la luz. Es preciso saber que la Tierra gira alrededor del Sol y que la Luna no emite luz propia sino que refleja la solar, que rota sobre su eje, que gira alrededor de la Tierra y que se traslada con ella alrededor del Sol. También es necesario saber que la Tierra rota para comprender por qué la Luna sólo es visible en determinadas ocasiones. Y después de interiorizar todos estos fenómenos y comprender la estructura del Sistema Solar, el alumnado en muchos casos aún posee concepciones que le hacen pensar que la explicación del movimiento de los cuerpos en la Tierra y sus proximidades es distinta a la de los cuerpos muy alejados de ella, manteniendo todavía, en alguna medida, una diferencia entre el mundo celeste y terrestre (LANCIANO, 1989).

Además de estas dificultades se han señalado otras problemáticas añadidas. Autores como Schoon (1995), Trundle, Atwood y Christopher (2002) o Kikas (2004) afirman que las propias concepciones alternativas del profesorado sobre estas cuestiones pueden ser uno de los orígenes de las ideas del alumnado. En la enseñanza de la Astronomía se han encontrado errores conceptuales en profesorado de diferentes niveles educativos y persistentes ideas alternativas, como muestran estudios de diferentes contextos, como Rutherford (2004) en EEUU, Trumper (2003) en Israel, Dai y Capie (1990) en Taiwan o Vega (2001) en España. Algunos estudios muestran incluso niveles de competencia entre el profesorado semejantes a los identificados 
en la población infantil (ATWOOD; ATWOOD, 1995; HOPE; TOWNSEND, 1983). Tal y como defienden Fenández y Peña (2007), las deficiencias disciplinares del profesorado tienen consecuencias en sus prácticas educativas; si estas personas presentan concepciones alternativas sobre los contenidos que deben trabajar con su alumnado, difícilmente podrán servir de guía. También recalcan que para que el profesorado tenga en cuenta las concepciones alternativas de su alumnado, primero debe ser consciente de sus propias concepciones sobre los contenidos en cuestión. Otro factor influyente en la generación de estas ideas también parecen ser los recursos utilizados en la enseñanza de la Astronomía. Así, se han estudiado los errores presentes en algunos recursos educativos. Pérez, Álvarez y Serrallé (2009) identificaron numerosas incorrecciones que contienen libros de texto españoles de enseñanza secundaria relacionados con la evolución histórica del conocimiento del Universo. También Kriner (2004) apunta algunos errores contenidos en textos educativos argentinos en relación con las fases de la Luna. De esta forma, aunque tanto el profesorado como los recursos deberían ser una fuente fiable para la enseñanza de la Astronomía, estas evidencias indican que pueden actuar como reforzador para las concepciones alternativas del alumnado.

\section{Objetivo}

El objetivo de este estudio es indagar sobre ideas alternativas frecuentes relacionadas con aspectos astronómicos de profesorado en formación, investigando si en el marco español aparecen los mismos esquemas alternativos que en otros contextos.

\section{Metodología}

\section{Instrumento de estudio}

En el estudio de las concepciones alternativas en Astronomía se ha utilizado una combinación de técnicas cuantitativas y cualitativas de recolección de datos. Se han empleado entrevistas individuales en combinación con preguntas escritas abiertas (ATWOOD; ATWOOD, 1995; ATWOOD; ATWOOD, 1996; CAMINO 1995; MANUEL, 1995); cuestionarios sólo con preguntas abiertas cuya contestación requiere redactar una explicación y realizar dibujos o esquemas (NAVARRETE, 1998; PARKER; HEYWOOD, 1998; VEGA, 2001) o cuestionarios de selección múltiple (RUTHERFORD, 2004; SCHOON, 1995; TRUMPER, 2003; ZEILIK; SCHAU; MATTERN, 1998). En muchas ocasiones las técnicas cualitativas sirvieron como instrumento de aproximación al tema, creándose después cuestionarios que incluían las concepciones alternativas más frecuentes y realizando análisis cuantitativos.

En este trabajo se optó por realizar un estudio cuantitativo empleando un cuestionario de opción múltiple (en el futuro se pretende continuar la investigación con una combinación de técnicas cuantitativas y cualitativas). Tras la realización de una extensiva revisión bibliográfica sobre cuestionarios relacionados con contenidos astronómicos, se seleccionaron diez preguntas en las que era frecuente que se hicieran patentes concepciones alternativas del alumnado. Las preguntas se redactaron respetando las fuentes originales, con la excepción de la inclusión 
de una opción de respuesta adicional en una pregunta (la número 9, que se refiere a la causa de la estaciones). El cuestionario está siendo utilizado en otras investigaciones del grupo (por ejemplo VARELA et al., 2013).

Las ventajas de utilizar preguntas ya incluidas en otros ensayos son numerosas. En primer lugar, estas preguntas ya han sido probadas, y las opciones de respuesta que incluyen no han sido seleccionadas al azar, sino que, en su mayoría, se corresponden con ideas alternativas frecuentes. Otra ventaja de utilizar preguntas ya empleadas en otras investigaciones es que permiten realizar una comparación entre los resultados de estudios realizados en marcos diferentes. Muchas de las investigaciones referidas a este tema proceden del ámbito anglosajón. En Estados Unidos, Lightman y Sadler (1993) estudiaron las concepciones alternativas sobre Astronomía en estudiantes de Secundaria. Schoon (1995), en el mismo país, lo hizo con estudiantes de magisterio, Zeilik, Schau y Mattern (1998) con estudiantes universitarios y Rutherford (2004) con distintos grupos de educadores formales y no formales. Fuera de este ámbito Trumper (2000, 2001, 2003), en Israel, trabajó con alumnado universitario, incluyendo el análisis de las ideas alternativas sobre Astronomía de futuro profesorado de Primaria y de Secundaria. En Estonia, Kikas (2004) estudió, en alumnado y profesorado de primaria, las concepciones sobre diversos tópicos científicos, entre los que se encontraban aspectos astronómicos. Camino (1995), en Argentina, investigó las diferentes concepciones alternativas más frecuentes entre profesorado de Primaria con un cuestionario de preguntas abiertas sobre el día y la noche, las estaciones o las fases de la Luna. También en el mismo país, Kriner (2004) estudió las ideas sobre las fases de la Luna en personas en edad infantil y adulta. Y en otros países sudamericanos se realizaron estudios similares, como el de Fernández y Peña (2007) en Méjico.

\section{Descripción de la muestra}

El estudio se realizó tomando como muestra alumnado universitario del Grado en Educación Primaria y del Grado en Educación Infantil de la Facultad de Ciencias de la Educación y del Deporte de Pontevedra, perteneciente a la Universidad de Vigo.

En total fueron encuestadas 145 personas, de las cuales 119 eran mujeres y 23 hombres (3 personas no respondieron la pregunta relativa al sexo). 84 pertenecían al Grado de Educación Primaria (58\%) y 61 al Grado de Educación Infantil (42\%). El rango de edades varía entre 19 y 39 años, siendo el 90\% menor de 30 años y la media de edad de 22,4 años. En cuanto a su formación, la mayor parte de este alumnado provenía del bachillerato de Humanidades y Ciencias Sociales (50\%), pero también de los bachilleratos de Ciencias y Tecnología (32\%) y Artes (3\%), así como de otros estudios $(10 \%)$.

\section{Análisis realizado}

Se elaboraron tablas de frecuencia de respuesta en cada pregunta con el propósito de realizar un análisis estadístico inferencial de los resultados. Se halló la proporción de respuestas correctas, así como la media y mediana de respuestas correctas por estudiante. Asimismo se agruparon los datos en diferentes grupos para facilitar su análisis comparativo y conocer los niveles de significatividad de las diferencias encontradas. 
El análisis se realizó mediante pruebas no paramétricas, por las siguientes razones: (a) la muestra no cumple los supuestos de normalidad y homecedasticidad que requieren las pruebas paramétricas y (b) el tamaño de los grupos que se comparan no es lo suficientemente grande en todos los casos. Así, se realizó un análisis de contraste mediante el test $U$ de Mann-Whitney y se utilizó la prueba de probabilidad exacta de Fisher para valorar la influencia significativa de las distintas variables en los datos encontrados. Esta prueba permite el análisis de datos nominales y es apropiada para resultados que no cumplen las condiciones de frecuencia esperada. De esta forma se hizo un análisis comparativo de los datos teniendo en cuenta distintas variables: género, bachillerato de origen y titulación, trabajando siempre con un nivel de confianza del 95\%. El análisis de los datos se realizó con el programa SPSS versión 20 para Windows.

\section{Resultados}

\section{Análisis de cada cuestión}

(1) Pregunta 1 del cuestionario

\section{1) ¿Qué causa las fases de la Luna?}

$\square$ a. Algo pasa por delante de la luna

$\square$ b. La Luna se mueve dentro y fuera de la sombra de la Tierra

$\square$ c. La Luna se mueve dentro y fuera de la sombra del Sol

$\square$ d. El otro lado de la Luna está siempre oscuro y es el que vemos

$\square$ e. Ninguna de las anteriores

En la primera pregunta sólo el 31\% de los encuestados ha elegido la opción adecuada (ninguna de las respuestas es correcta), ya que las fases de la Luna se deben a su movimiento alrededor de la Tierra y a la reflexión de los rayos solares sobre ella. En esta cuestión Zeilik, Schau y Mattern (1998), con alumnado universitario, y Lightman y Sadler (1993), con alumnado de secundaria, obtuvieron valores en torno al 25\%, aunque otros como Rutherford (2004) rebajan esta cifra hasta el $12 \%$ trabajando con distintos tipos de educadores. La mayor parte del estudiantado (36\%) ha escogido la respuesta que relaciona las fases de la Luna con su movimiento dentro y fuera de la sombra de la Tierra. Esta idea es frecuente, tal y como recogen los estudios de Trumper (2001) o Rutherford (2004). Varios estudios (RUTHERFORD, 2004; ZEILIK; SCHAU; MATTERN, 1998) señalan que las concepciones alternativas referentes a esta cuestión presentan una gran persistencia.

(2) Pregunta 2 del cuestionario

2) ¿Cuántas veces está el sol al mediodía directamente sobre tu cabeza?

$\square$ a. Todos los días

$\square$ b. Sólo en el verano

$\square$ c. Sólo la semana del solsticio de verano

$\square$ d. Sólo un día al año

$\square$ e. Nunca 
En la segunda pregunta menos del 10\% de las personas encuestadas ha respondido correctamente, indicando que el Sol nunca se encuentra directamente sobre su cabeza (en la latitud en la que se aplicó el cuestionario, por encima del Trópico de Cáncer, nunca se produce esta situación). En dicha cuestión Zeilik, Schau y Mattern (1998) obtuvieron, con alumnado universitario, un $23 \%$ de respuestas correctas, y Trumper (2000) un 32\%. La mayor parte del alumnado (41\%) escogió la opción "Todos los días", idea alternativa que es muy frecuente, tal y como muestran los estudios de Rutherford (2004) y Trumper (2001), que obtuvieron resultados similares a estos, o Schoon (1995), que halló una presencia del 67\% entre alumnado de magisterio. Otra idea frecuente $(20 \%$ de las respuestas) es que este fenómeno ocurre una vez al año, probablemente relacionándolo con el solsticio de verano.

(3) Pregunta 3 del cuestionario

3) Para que ocurra un eclipse total de sol, ¿en qué fase debe estar la luna?

$\square$ a. luna llena

$\square$ b. luna menguante

$\square$ c. luna nueva

$\square$ d. luna creciente

De nuevo, en la tercera pregunta, pocas personas han escogido la respuesta correcta. Sólo el 14\% cree que para que ocurra un eclipse total de Sol la fase debe ser Luna nueva. Otros autores como Zeilik, Schau y Mattern (1998) obtuvieron un 28\%, y Trumper (2001) un 22\%. La mayoría de las personas (un 78\%) asocia el eclipse total de Sol con la Luna llena. Los autores previamente citados obtuvieron asimismo valores similares para esta opción de respuesta, y por ejemplo Trumper (2001) halló valores superiores al 70\%.

(4) Pregunta 4 del cuestionario

4) La Tierra ejerce una fuerza gravitatoria sobre la Luna. Pero la Luna ¿ejerce una fuerza gravitatoria sobre la Tierra?

$\square$ a. Sí, pero menor

$\square$ b. Sí, igual

$\square$ c. Sí, pero mayor

$\square$ d. No, la Luna no tiene gravedad

En la cuarta pregunta solo un $16 \%$ ha respondido correctamente, indicando que la Luna ejerce sobre la Tierra una fuerza gravitacional igual a la que nuestro planeta produce sobre ella. Resultados semejantes fueron obtenidos por Rutherford (2004) con educadores (18\%) o Zeilik, Schau y Mattern (1998) con alumnado universitario (12\%). La mayoría de las personas encuestadas en el presente estudio piensa que la Luna produce una fuerza gravitatoria menor que la Tierra (58\%) o incluso que no tiene gravedad (21\%). También Rutherford (2004) obtuvo, como idea alternativa más frecuente, que la Luna no tiene gravedad $(29 \%)$. 
(5) Pregunta 5 del cuestionario

5) Al observar la Luna desde la Tierra, siempre vemos la misma cara. Esta observación significa que la Luna...

$\square$ a. No gira sobre su eje

$\square$ b. Gira sobre su eje una vez al día

$\square$ c. Gira sobre su eje una vez al mes

$\square$ d. Gira sobre su eje una vez al año

En la quinta cuestión, cuando se pregunta por qué siempre es visible la misma cara de la Luna sólo el 14\% de las personas elige la respuesta adecuada, que la Luna gira sobre su eje aproximadamente - una vez al mes. Otras investigaciones también han hallado porcentajes de éxito bajos en esta cuestión. Trumper (2001) con alumnado universitario obtuvo valores de éxito algo superiores (22\%), pero otros como Zeilik, Schau y Mattern (1998) o Rutherford (2004) obtuvieron datos inferiores al 15\%. Así, la mayor parte de las personas responde incorrectamente. Un 29\% afirma que la Luna gira sobre sí misma una vez al día y más de la mitad (51\%) defiende que no gira sobre su eje. Trumper (2001) y Rutherford (2004) encuentran también valores en torno al 50\% sobre esta última idea alternativa.

(6) Pregunta 6 del cuestionario

6) ¿Qué causa el día y la noche?

$\square$ a. La Tierra gira sobre su eje

$\square$ b. La Tierra se mueve alrededor del Sol.

$\square$ c. Las nubes bloquean la luz del Sol

$\square$ d. El Sol gira alrededor de la Tierra

$\square$ e. La Tierra se mueve dentro y fuera de la sombra del sol

En la sexta cuestión, el 69\% de las personas atribuye correctamente a la rotación terrestre la existencia de días y noches. En la mayor parte de los estudios el porcentaje de éxito en esta pregunta es elevado: Trumper (2001) obtuvo un 62\%, Rutherford (2004) un 65\% y Schoon (1995) un 77\%. En la presente investigación también aparece un 25\% de personas que responde incorrectamente, defendiendo que esta sucesión de días y noches se debe a que la Tierra se mueve alrededor del Sol. Esta es la idea alternativa más frecuente en este estudio, así como en los de Trumper (2001) y Schoon (1995).

(7) Pregunta 7 del cuestionario

7) La Luna gira alrededor de la Tierra en...

$\square$ a. una hora

$\square$ b. un día

$\square$ c. una semana

$\square$ d. un mes

$\square$ e. un año 
En la séptima cuestión, sólo el 37\% contesta correctamente (la Luna gira alrededor de la Tierra en un aproximadamente un mes). La mayoría de las personas encuestadas (43\%) piensa que tarda sólo un día en realizar este recorrido y un $11 \%$ piensa que lo hace en un año. Rutherford (2004) obtuvo un 42\% de respuestas correctas y Trumper (2001) un 60\%. La respuesta alternativa más frecuente (un día) es elegida por un 43\% de la muestra. Rutherford (2004) obtiene en esta misma opción de respuesta un $42 \%$ de frecuencia.

(8) Pregunta 8 del cuestionario

8) De acuerdo con las ideas y observaciones actuales, ¿¿cuál de las siguientes afirmaciones es correcta?

$\square$ a. La Tierra es el centro del universo

$\square$ b. El Sol es el centro del universo

$\square$ c. La Vía Láctea es el centro del universo

$\square$ d. El universo no tiene un centro

A la octava pregunta el $70 \%$ del alumnado responde correctamente, opinando que el universo no tiene centro. Es la cuestión con mayor índice de acierto. En la muchos otros estudios se obtuvo también un porcentaje de éxito elevado en esta pregunta, tal y como muestra Trumper (2001), quien obtiene un 73\%. Sin embargo, un 23\% de personas piensa que el Sol es el centro del universo, e incluso un $4 \%$ que opina que dicho centro es la Tierra. Rutherford (2004) obtiene en esta cuestión resultados diferentes, con un 59\% de personas defendiendo esta última teoría.

(9) Pregunta 9 del cuestionario

9) Las diferentes estaciones que experimentamos todos los años se deben a...

$\square$ a. La distancia que varía entre el Sol y la Tierra

$\square$ b. Las distancias que varían entre el Sol, la Tierra y la Luna

$\square$ c. La inclinación del eje de la Tierra que gira alrededor del Sol.

$\square$ d. Diversos grados de contaminación atmosférica que diluyen los rayos del Sol

$\square$ e. La variedad de climas que existen en la Tierra

En la novena pregunta, el 64\% responde correctamente, señalando que las estaciones se deben a la inclinación constante del terrestre durante su movimiento de traslación. Los estudios que tratan este tema obtienen resultados que difieren entre sí, con un 10\% de respuestas correctas halladas por Lightman y Sadler (1993) con estudiantes de secundaria, un 41\% de Rutherford (2004) con educadores o un 67\% de Trumper (2001) con alumnado universitario. En el presente estudio aparece asimismo un porcentaje importante de personas (29\%) que responde que las estaciones son causadas por la variación de la distancia entre el Sol y la Tierra. Esta es la idea alternativa más frecuente también en los estudios de Trumper (2001) y Rutherford (2004). 
(10) Pregunta 10 del cuestionario

10. ¿Cuál de las siguientes cuatro diagramas describe con mayor precisión la forma de la órbita de la Tierra alrededor del Sol?

\begin{tabular}{cccc}
\hline & B & C & D \\
\hline+8 & &
\end{tabular}

En la última cuestión sólo el 6\% de las personas responde correctamente. La mayoría $(63 \%)$ indica que el diagrama que describe con mayor precisión la forma de la órbita terrestre es el que presenta una órbita elíptica muy excéntrica con el Sol situado en uno de los focos. La respuesta correcta es, sin embargo, la opción "a", ya que la órbita terrestre es casi circular (la variación de la distancia al centro varía como máximo en un 1,39\%) y el Sol está situado prácticamente en su centro. Tal y como explica Rutherford (2004), esta idea a menudo tiene su origen en la aparición de órbitas terrestres muy excéntricas en los libros de texto elementales (IVOWI; OLUDOTUN, 1987). Así, en este estudio, esta idea errónea de la órbita terrestre es la concepción alternativa más frecuente, que defiende más del $90 \%$ de los estudiantes.

\section{Análisis global de los datos}

En el análisis global de los datos (Figura 1), se obtuvo un porcentaje promedio de acierto del $33 \%$. Autores que también indagaron sobre las concepciones alternativas sobre Astronomía en alumnado universitario, aunque con diferentes cuestionarios, como Trumper (2000) o Zeilik, Schau y Mattern (1998) obtuvieron porcentajes del 48\% y del 40\%, respectivamente. Cabe señalar que en la presente investigación sólo un 1\% del alumnado obtuvo siete de diez respuestas correctas, el máximo obtenido, y que el 3\% erró en todas las preguntas. Tal y como se muestra en la Figura 2, las cuestiones con mayor porcentaje de éxito fueron las relativas: (a) al centro del Universo (P8), con un 70\%; (b) a la causa del día y la noche (P6), con un 69\%, y; (c) a la razón de la variabilidad estacional (P9), con un 64\%.

Los peores resultados de la prueba, con un nivel de éxito del $6 \%$, fueron obtenidos en la última cuestión sobre la forma de la órbita terrestre alrededor del Sol. Aparecen también otras cuatro preguntas con niveles de éxito inferiores al 20\%, y son relativas a la posición del Sol al mediodía (P2) con un 10\%, a la fase de la Luna en un eclipse solar (P3) con un 14\%, a la rotación lunar (P5) con otro 14\%, y a la gravedad lunar (P4) con un 16\%. 
Figura 1. Porcentajes de éxito en cada pregunta, en función de las distintas variables estudiadas

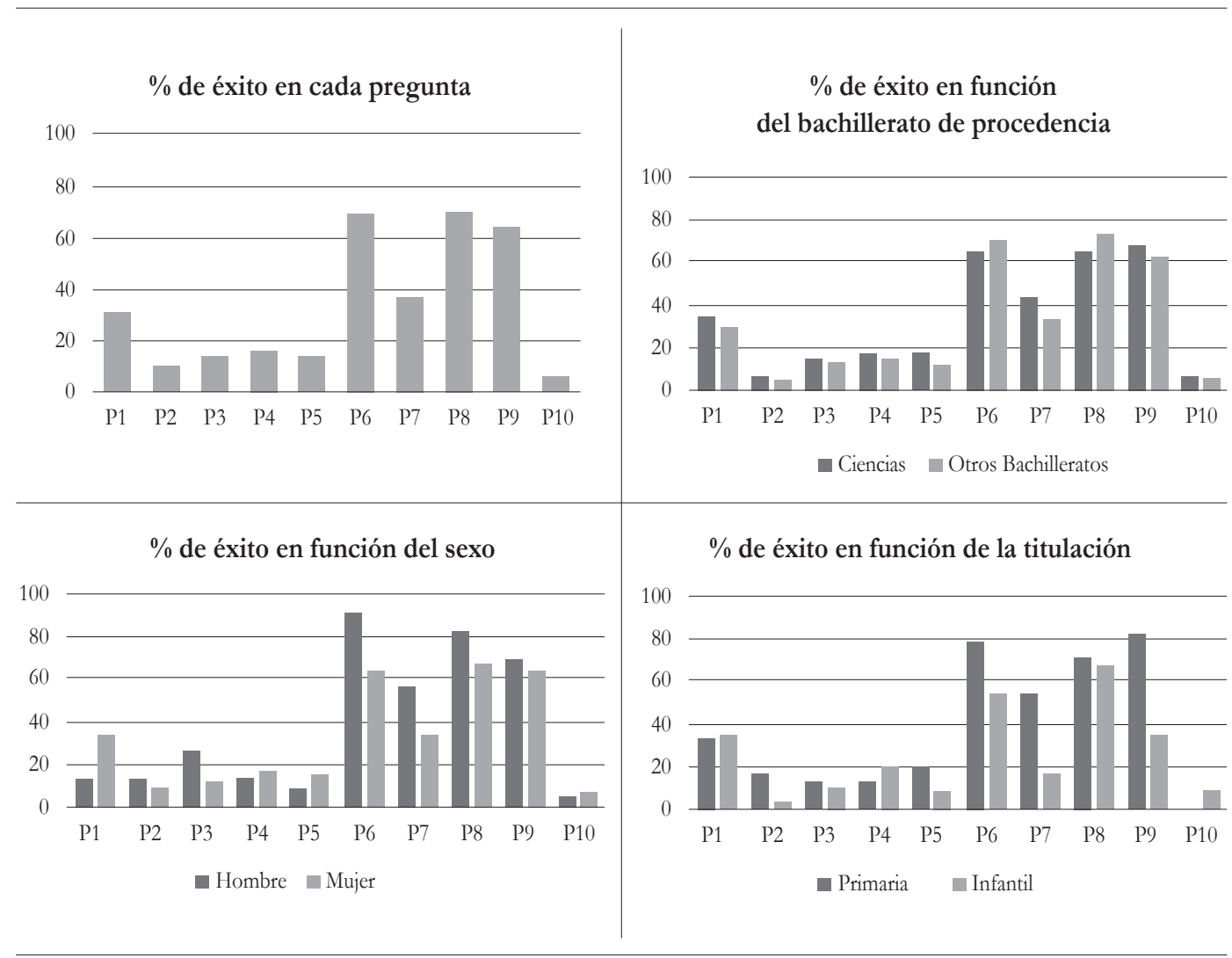

Fuente: elaboración de los autores.

Cuando se compararon los datos obtenidos en función del bachillerato de procedencia de los estudiantes (Tabla 1), se observó que el nivel de éxito en alumnado que proviene de un bachillerato de Ciencias (34\%) es similar al del que proviene de otros (33\%). Así, cuando se compara el número de respuestas correctas por persona de ambos grupos mediante el test de Mann-Whitney (Tabla 1) se obtiene que no son significativamente diferentes ( $\leq \leq 0,05)$. Otros autores, como Schoon (1988) tampoco encontraron diferencias significativas entre el alumnado que había cursado asignaturas de ciencias experimentales, ya sea en la universidad o en la escuela secundaria, y aquel que no tenía dicha formación. De hecho, cuando se analizan los datos pregunta a pregunta, no se obtiene en ninguna de ellas una relación significativa $(p \leq 0,05)$ entre el bachillerato cursado y el éxito alcanzado al responder la cuestión.

En cuanto a diferencias de género, los porcentajes de acierto obtenidos fueron en hombres de un $38 \%$ y en mujeres de un $32 \%$, pero estas diferencias no resultan significativas cuando se realiza una comparación mediante el test de Mann-Whitney (Tabla 1). Otros autores sí han encontrado diferencias de género significativas en estudios similares (TRUMPER, 2000). Sin embargo, Bisard et al. (1994), que investigaron la persistencia de ideas alternativas en temas 
relacionados con la Física y las Ciencias de la Tierra, encontraron que las diferencias de género disminuían al aumentar el nivel educativo, hasta ser prácticamente inexistentes en los niveles superiores. En un análisis pormenorizado de cada pregunta del presente estudio mediante el test de Fisher se obtuvieron diferencias significativas $(\mathrm{p} \leq 0,05)$ con respecto al género (Tabla 2) sólo en una cuestión (P6). Cuando se pregunta por la causa del día y la noche, los hombres obtuvieron un mayor nivel de acierto que las mujeres.

Al realizar la comparativa en función de la titulación el número de respuestas correctas por persona de ambos grupos es significativamente diferente (Tabla 3). Mientras el alumnado de $2^{\circ}$ del Grado en Educación Primaria presenta un porcentaje de éxito del 38\%, el de $2^{\circ}$ del Grado en Educación Infantil presenta un porcentaje del 26\%. De hecho, en el análisis aparecen diferencias significativas en función de la titulación en cuatro preguntas. Cuando se pregunta cuántas veces está el sol sobre tu cabeza (P2) el alumnado del Grado en Educación Primaria obtiene porcentajes de éxito significativamente mayores. Ocurre lo mismo cuando se pregunta por la causa del día y la noche (P6), por el tiempo que tarda la Luna en girar alrededor de la Tierra, o por la causa de las estaciones (P9).

Tabla 1. Análisis de contraste (número de respuestas correctas) mediante el test U de Mann-Whitney.

\begin{tabular}{|c|c|c|c|c|}
\hline & Éxito & $\mathbf{N}$ & Estadísticos de co & traste \\
\hline $\begin{array}{l}\text { Bachillerato } \\
\text { Ciencias } \\
\text { Otros }\end{array}$ & $\begin{array}{l}34 \% \\
33 \%\end{array}$ & $\begin{array}{l}(\mathrm{N}=46) \\
(\mathrm{N}=99)\end{array}$ & $\begin{array}{l}\text { U de Mann-Whitney } \\
\text { W de Wilcoxon } \\
\text { Z } \\
\text { Sig. asintót. (bilateral) }\end{array}$ & $\begin{array}{r}2179,000 \\
7129,000 \\
-, 424 \\
, 672\end{array}$ \\
\hline $\begin{array}{l}\text { Sexo } \\
\text { Hombre } \\
\text { Mujer }\end{array}$ & $\begin{array}{l}38 \% \\
32 \%\end{array}$ & $\begin{array}{l}(\mathrm{N}=23) \\
(\mathrm{N}=119)\end{array}$ & $\begin{array}{l}\text { U de Mann-Whitney } \\
\text { W de Wilcoxon } \\
\text { Z } \\
\text { Sig. asintót. (bilateral) }\end{array}$ & $\begin{array}{r}1086,500 \\
8226,500 \\
-1,590 \\
, 112\end{array}$ \\
\hline $\begin{array}{l}\text { Titulación } \\
\text { Primaria } \\
\text { Infantil }\end{array}$ & $\begin{array}{l}38 \% \\
26 \%\end{array}$ & $\begin{array}{l}(\mathrm{N}=57) \\
(\mathrm{N}=61)\end{array}$ & $\begin{array}{l}\text { U de Mann-Whitney } \\
\text { W de Wilcoxon } \\
\text { Z } \\
\text { Sig. asintót. (bilateral) }\end{array}$ & $\begin{array}{r}929,500 \\
2820,500 \\
-4,443 \\
, 000\end{array}$ \\
\hline
\end{tabular}

Fuente: elaboración de los autores.

Tabla 2. Análisis de significatividad (Estadístico exacto de Fisher), donde N=142.

\begin{tabular}{llccc}
\hline & Respuestas & Hombre & Mujer & Sig. exacta (bilateral) \\
\hline \multirow{2}{*}{ Día y noche (P6) } & Correctas & 21 & 76 &, 012 \\
& Incorrectas & 2 & 43 &, \\
\hline
\end{tabular}

Fuente: elaboración de los autores. 
Tabla 3. Análisis de significatividad (Estadístico exacto de Fisher), donde N=118.

\begin{tabular}{|c|c|c|c|c|}
\hline & Respuestas & Primaria & Infantil & Sig. exacta (bilateral) \\
\hline \multirow[t]{2}{*}{ Sol al mediodía sobre tu cabeza (P2) } & Correctas & 9 & 2 & 026 \\
\hline & Incorrectas & 48 & 59 & \\
\hline \multirow{2}{*}{ Día y noche (P6) } & Correctas & 45 & 33 & ,006 \\
\hline & Incorrectas & 12 & 28 & \\
\hline \multirow[t]{2}{*}{ Período lunar alrededor de la Tierra (P7) } & Correctas & 31 & 10 & ,000 \\
\hline & Incorrectas & 26 & 51 & \\
\hline \multirow[t]{2}{*}{ Causa de las estaciones (P9) } & Correctas & 47 & 21 & ,000 \\
\hline & Incorrectas & 10 & 40 & \\
\hline
\end{tabular}

Fuente: elaboración de los autores.

\section{Discusión y conclusiones}

Los resultados obtenidos no difieren mucho de los hallados en otras investigaciones (RUTHERFORD 2004; SCHOON, 1995; TRUMPER, 2001; ZEILIK; SCHAU; MATTERN, 1998), encontrándose aquí concepciones alternativas que también han quedado patentes en otros estudios (ver Tabla 4):

1. El Sol se encuentra todos los días sobre su cabeza al mediodía (o un día al año).

2. Las fases de la Luna son causadas porque ésta entra y sale de la sombra de la Tierra (o del Sol).

3. Para que ocurra un eclipse solar la Luna debe estar llena.

4. La Luna ejerce una fuerza gravitatoria sobre la Tierra menor que ésta sobre su satélite (o la Luna no tiene gravedad).

5. La Luna no gira sobre su eje.

6. El día y la noche son causados porque la Tierra se mueve alrededor del Sol.

7. La Luna gira alrededor de la Tierra en un día.

8. El Sol es el centro del universo.

9. Las estaciones se producen por las variaciones de distancia entre el Sol y la Tierra.

10. La órbita terrestre tiene una forma claramente elíptica.

La idea alternativa más frecuente hallada en este estudio está relacionada con la forma de la orbita terrestre - un 85\% defiende esquemas claramente elípticos. Conviene también destacar los problemas que el alumnado tiene para describir los fenómenos relacionados con los movimientos de la Luna, tal y como indica que el $79 \%$ piense que un eclipse total de Sol se produce con Luna llena, el 51\% señale que ésta no tiene rotación y el 43\% defienda que el satélite gira alrededor de la Tierra en un día. También es importante la proporción de alumnado (58\%) que cree que la Luna ejerce una fuerza gravitatoria sobre la Tierra menor que ésta sobre su satélite.

Por otra parte, el porcentaje total de respuestas correctas en el cuestionario utilizado es de sólo un 33\%. El análisis de la influencia de distintas variables no permite concluir que el bachillerato de procedencia pueda tener influencia en el éxito a la hora de responder, indi- 
Tabla 4. Porcentaje de acierto en cada pregunta e ideas alternativas más frecuentes.

\begin{tabular}{|c|c|c|c|c|}
\hline Ítem & $\begin{array}{l}\% \text { de } \\
\text { éxito }\end{array}$ & $\begin{array}{l}\text { Idea alternativa } \\
\text { más frecuente }\end{array}$ & $\begin{array}{l}\quad \% \text { de } \\
\text { respuesta } \\
\text { aternativa }\end{array}$ & $\begin{array}{c}\% \text { de éxito } \\
\text { en otros estudios }\end{array}$ \\
\hline $\begin{array}{l}\text { (1) Posición del Sol } \\
\text { directamente sobre tu cabeza } \\
\text { al mediodía }\end{array}$ & 31 & Todos los días & 41 & $\begin{array}{l}\text { Zeilik; Schau; Mattern } \\
(1998)-23 \% \\
\text { Lightman; Sadler (1993)-22\% } \\
\text { Rutherford (2004) - } 12 \%\end{array}$ \\
\hline $\begin{array}{l}\text { (2) Causa de las fases de la } \\
\text { Luna }\end{array}$ & 10 & $\begin{array}{l}\text { La Luna se mueve } \\
\text { dentro y fuera de la } \\
\text { sombra de la Tierra }\end{array}$ & 36 & $\begin{array}{l}\text { Zeilik; Schau; Mattern } \\
(1998)-23 \% \\
\text { Trumper }(2000)-32 \%\end{array}$ \\
\hline $\begin{array}{l}\text { (3) Fase lunar durante un } \\
\text { Eclipse total de Sol }\end{array}$ & 14 & Luna llena & 79 & $\begin{array}{l}\text { Zeilik; Schau; Mattern } \\
(1998)-28 \% \\
\text { Trumper }(2001)-22 \%\end{array}$ \\
\hline $\begin{array}{l}\text { (4) Fuerza gravitatoria lunar } \\
\text { sobre la Tierra }\end{array}$ & 16 & $\begin{array}{l}\text { Menor que la } \\
\text { terrestre }\end{array}$ & 58 & $\begin{array}{l}\text { Zeilik; Schau; Mattern } \\
(1998)-12 \% \\
\text { Rutherford (2004) - 18\% }\end{array}$ \\
\hline (5) Rotación lunar & 14 & No gira sobre su eje & 51 & $\begin{array}{l}\text { Zeilik; Schau; Mattern } \\
(1998)-10 \% \\
\text { Trumper }(2001)-22 \%\end{array}$ \\
\hline (6) Causa del día y la noche & 69 & $\begin{array}{l}\text { La Tierra se mueve } \\
\text { alrededor del Sol }\end{array}$ & 25 & $\begin{array}{l}\text { Schoon }(1995)-77 \% \\
\text { Trumper }(2001)-62 \% \\
\text { Rutherford (2004) - 65\% }\end{array}$ \\
\hline $\begin{array}{l}\text { (7) Movimiento lunar } \\
\text { alrededor de la Tierra }\end{array}$ & 37 & Un día & 43 & $\begin{array}{l}\text { Trumper }(2001)-60 \% \\
\text { Rutherford (2004) }-42 \%\end{array}$ \\
\hline (8) Centro del universo & 70 & El Sol & 23 & $\begin{array}{l}\text { Trumper (2001) - 73\% } \\
\text { Rutherford (2004) - 59\% }\end{array}$ \\
\hline (9) Causa de las estaciones & 64 & $\begin{array}{l}\text { La distancia que } \\
\text { varía entre el Sol y la } \\
\text { Tierra }\end{array}$ & 29 & $\begin{array}{l}\text { Lightman; Sadler (1993) - 10\% } \\
\text { Trumper (2001) - 67\% } \\
\text { Rutherford (2004) - 41\% }\end{array}$ \\
\hline $\begin{array}{l}\text { (10) Forma más precisa de } \\
\text { la órbita terrestre alrededor } \\
\text { del Sol }\end{array}$ & 6 & & 63 & \\
\hline
\end{tabular}

Fuente: elaboración de los autores.

cando los datos que no existen diferencias significativas entre el alumnado que proviene de un bachillerato de Ciencias o de otros. Sin embargo, sí aparecen diferencias significativas cuando se estudia la influencia del grado cursado. El profesorado en formación perteneciente al Grado en Educación Primaria obtiene mejores porcentajes de éxito que el de Educación Infantil, y aparecen diferencias significativas en 4 de las 10 cuestiones. Con respecto al sexo, los hombres obtienen una media de éxito ligeramente superior, que no es significativa, y sólo en una pregunta aparecen diferencias importantes. 
Los resultados de este estudio parecen señalar la necesidad de una mejora en el proceso de enseñanza y aprendizaje de los contenidos relacionados con la Astronomía, utilizando, en mayor medida, otros recursos y metodologías más adecuados, trabajando de manera interdisciplinar desde diferentes áreas o asignaturas, aprovechando las oportunidades que ofrecen los cursos de formación inicial y continua de profesorado... Aunque, como señalan Monereo et al. (2009), para promover cambios reales en las prácticas del profesorado no basta con introducir nuevos recursos, materiales o técnicas: un cambio significativo en las formas de actuación requiere un cambio sustancial en las concepciones. De esta forma, también parece importante replantearse la formación básica del profesorado, que, tal vez, debería propiciar el análisis de sus propias ideas alternativas y de las consecuencias que esto produce sobre su práctica educativa. En este sentido, conviene recordar aquí las palabras de Rosa M. Pujol:

El objetivo de la educación científica no es cambiar las concepciones alternativas de los escolares por concepciones científicas. El objetivo es contribuir a que los escolares vean que existen distintos puntos de vista que permiten explicar una misma entidad y que el de la ciencia y el de ellos poseen características distintas. [...] Se trata de ayudarles a diferenciar entre sus razonamientos y las expresiones de los mismos y los que utiliza la ciencia, así como de ayudarles a entender el porqué de dichas diferencias. (PUJOL, 2003, p. 94-95)

De esta forma, dentro de un marco socioconstructivista (IZQUIERDO, 2001), el trabajo con las concepciones alternativas del alumnado, propiciando que éste haga explícitos sus modelos de pensamiento y creando situaciones que le hagan cuestionarse sus modelos, puede servir de ayuda en sus procesos de construcción del conocimiento.

Teniendo en cuenta todo lo antedicho, se abren nuevas líneas de investigación que el equipo pretende explorar. En este sentido, resulta imprescindible la realización de un estudio cualitativo que explore a fondo estas concepciones alternativas y su origen. Asimismo se está proyectando investigar acerca de posibles intervenciones educativas en la línea de las apuntadas previamente.

\section{Agradecimientos}

Al proyecto EDU2012-38022-C02-01 sobre el desarrollo de prácticas y competencias financiado por el Ministerio de Economía y Competitividad. 


\section{Referencias}

ATWOOD, V. A.; ATWOOD, R. K. Preservice elementary teachers' conceptions of what causes night and day. School Science and Mathematics, Hoboken, v. 95, n. 6, p. 290-294, 1995.

ATWOOD, R. K.; ATWOOD, V. A. Preservice elementary teachers' conceptions of the causes of seasons. Journal of Research in Science Teaching, Hoboken, v. 33, n. 5 , p. 553-563, 1996.

BISARD W. et al. Assessing selected physical science and earth science misconceptions of middle school through university preservice teachers. Journal of College Science Teaching, Arlington, v. 24, n. 1, p. 38-42, 1994.

BRETONES, P.; COMPIANI, M. Conceptual evolution of teachers about the daily motion of the celestial sphere. Ciência \& Educação, Bauru, v. 17, n. 3, p. 735-755, 2011.

CAMINO, N. Ideas previas y cambio conceptual en astronomía: un estudio con maestros de primaria sobre el día y la noche, las estaciones y las fases de la luna. Enseñanza de las Ciencias, Barcelona, v. 13, n. 1, p. 81-96, 1995.

DAI, M. F.; CAPIE, W. Misconceptions about the Moon held by preservice teachers in Taiwan. In: ANNUAL MEETING OF THE NATIONAL ASSOCIATION FOR RESEARCH IN SCIENCE TEACHING, 1990, Atlanta. Proceedings... Atlanta: NARST, 1990.

FERNÁNDEZ, M. T.; PEÑA, S. H. Concepciones de maestros de primaria sobre el día y la noche y las estaciones del año. Revista Latinoamericana de Estudios Educativos, México, v. 37, n. 3-4, p. 189-220, 2007.

GANGUI, A.; IGLESIAS, C.; QUINTEROS, C. Indagación llevada a cabo con docentes de primaria en formación sobre temas básicos de astronomía. Revista Electrónica de Enseñanza de las Ciencias, Vigo, v. 9, n. 2, p. 467-486, 2010.

GARCÍA, S.; MONDELO, M.; MARTÍNEZ, C. La astronomía en la formación de profesores. Alambique: didáctica de las ciencias experimentales, Barcelona, n. 10, p. 121-127, 1996.

HOPE, J.; TOWNSEND, M. Student teachers' understanding of science concept. Research in Science Education, Dordrecht, v. 13, n. 1, p. 177-184, 1983.

IACHEL, G.; LANGHI, R.; SCALVI, R. M. F. Concepções alternativas de alunos do ensino médio sobre o fenômeno de formação das fases da lua. Revista Latino-Americana de Educação em Astronomia, São Carlos, n. 5, p. 25-37, 2008.

IVOWI, U.; OLUDOTUN, J. An investigation of misconceptions in physics. In: NOVAK, J. D. (Ed.). Proceedings of the Second International Seminar Misconceptions and Educational Strategies in Science and Mathematics. Ithaca: Cornell University, 1987. $3 \mathrm{v}$. 
IZQUIERDO, M. Constructivismo versus socioconstructivismo. Enseñanza de las Ciencias de la Tierra, Madrid, v. 9, n. 3, p. 239-242, 2001.

KIKAS, E. Teachers' conceptions and misconceptions concerning three natural phenomena. Journal of Research in Science Teaching, Hoboken, v. 41, n. 5, p. 432-448, 2004.

KRINER, A. Las fases de la luna: ¿Cómo y cuándo enseñarlas? Ciência \& Educação, Bauru, v. 10, n, 1, p. 111-120, 2004.

LANCIANO, N. Ver y hablar como Tolomeo y pensar como Copérnico. Enseñanza de las Ciencias, Barcelona, v. 7, n. 2, p. 173-182, 1989.

LANGHI, R. Educação em astronomia: da revisão bibliográfica sobre concepções alternativas à necessidade de uma ação nacional. Caderno Brasileiro de Ensino de Física, Florianópolis, v. 28, n. 2, p. 373-399, 2011.

LANGHI, R.; NARDI, R. Ensino de astronomia: erros conceituais mais comuns presentes em livros didáticos de ciências. Caderno Brasileiro de Ensino de Física, Florianópolis, v. 24, n. 1, p. 87-111, abr. 2007.

. Formação de professores e seus saberes disciplinares em astronomia essencial nos anos iniciais do ensino fundamental. Ensaio: pesquisa em educação em ciências, Belo Horizonte, v. 12, n. 2, p. 205-224, maio-ago., 2010.

LIGHTMAN, A.; SADLER, P. M. Teacher predictions versus actual student gains. Physics Teacher, Melville, v. 31, n. 3, p. 162-167, 1993.

MANUEL, J. ¿Por qué hay veranos e inviernos?: representaciones de estudiantes (12-18) y de futuros maestros sobre algunos aspectos del modelo sol-tierra. Enseñanza de las Ciencias, Barcelona, v. 13, n. 2, p. 227-236, 1995.

MARTÍNEZ, J. M.; MARTÍNEZ, B. Preservice elementary teachers' conceptions of the Sun-Earth model: a proposal of a teaching-learning sequence. Astronomy Education Review, Tucson, v. 4, n. 1, p. 121-126, 2005.

MONEREO, C. et al. Las bases psicoeducativas del proyecto PISA como guía para el cambio en las concepciones y prácticas del profesorado de secundaria. Infancia y Aprendizaje, Abingdon, v. 32, n. 3, p. 421-447, 2009.

NAVARRETE, A. Una experiência de aprendizaje sobre los movimientos relativos del sistema "sol/tierra/luna" en el contexto de la formación inicial de maestros. Investigación en la Escuela, Sevilla, n. 35, p. 5-20, 1998.

. Obstáculos y dificultades en la evolución de las estructuras conceptuales y epistemológicas de los futuros maestros: un estudio de casos sobre el fenómeno de las estaciones. 2004. 744 f. (Tesis doctoral) - Departamento de Didáctica, Universidad de Cádiz, Cádiz, 2004. 
PARKER, J.; HEYWOOD, D. The Earth and beyond: developing primary teachers' understanding of basic astronomical events. International Journal of Science Education, Abingdon, v. 20, n. 5, p. 503-520, 1998.

PÉREZ, U.; ALVAREZ, M.; SERRALLÉ, F. Los errores de los libros de texto de primer curso de ESO sobre la evolución histórica del conocimiento del universo. Enseñanza de las Ciencias, Barcelona, v. 27, n. 1, p. 109-120, 2009.

PUJOL, R. M. Didáctica de las ciencias en la educación primaria. Madrid: Síntesis, 2003.

RUTHERFORD, L. Exploring alternative conceptions of teachers and informal educators about selected astronomy concepts. 2004. $152 \mathrm{f}$. Dissertation (Doctor in Education) - University of Cincinnati, Cincinnati, 2004. Disponible en: <https://etd. ohiolink.edu/!etd.send_file?accession=ucin1100986162\&disposition=inline $>$. Acceso el: 13 ago. 2015.

SANMARTÍ, N. Didáctica de las ciencias en la educación secundaria. Madrid: Síntesis, 2003.

SCHOON, K. J. Misconceptions in earth and space sciences: a cross-age study. 1988. (Ph.D. Dissertation) - Loyola University, Chicago, 1988.

. The origin and extent of alternative conceptions in the earth and space sciences: a survey of pre-service elementary teachers. Journal of Elementary Science Education, Macomb, v. 7, n. 2, p. 27-46, 1995.

TRUMPER, R. A cross-college age study of science and nonscience students' conceptions of basic astronomy concepts in preservice training for high school teachers. Journal of Science Education and Technology, Dordrecht, v. 10, n. 2, p. 189-195, 2001.

The need for change in elementary school teacher training: a cross-college age study of future teachers' conceptions of basic astronomy concepts. Teaching and Teacher Education, Oxford, UK, v. 19, n. 3, p. 309-323, 2003.

. University students' conceptions of basic astronomy concepts. Physics Education, Bristol, v. 35, n. 1, p. 9-15, 2000.

TRUNDLE, K. C.; ATWOOD, R.; CHRISTOPHER, J. E. Preservice elementary teachers' conceptions of Moon phases before and after instruction. Journal of Research in Science Teaching, Hoboken, v. 39, n. 7, p. 633-658, 2002.

VARELA, M. et al. Evolución de las concepciones sobre astronomía de profesorado en formación tras una intervención educativa con actividades de simulación. Enseñanza de las Ciencias, Barcelona, v. 31, p. 3612-3617, 2013. Número extra. 
VEGA, A. Tenerife tiene seguro de sol (y de luna): representaciones del profesorado de primaria acerca del día y la noche. Enseñanza de las Ciencias, Barcelona, v. 19, n. 1, p. 31-44, 2001.

Sol y luna, una pareja precopernicana: estudio del día y la noche en educación infantil. 2002. (Tesis doctoral) - Universidad de la Laguna, Tenerife, 2002.

ZEILIK, M.; SCHAU, C.; MATTERN, N. Misconceptions and their change in universitylevel astronomy courses. The Physics Teacher, Melville, v. 36, p. 104-107, 1998. 